\title{
Microbiota control immune regulation in humanized mice
}

\author{
Elke Gülden, ${ }^{1}$ Nalini K. Vudattu, ${ }^{1}$ Songyan Deng, ${ }^{1}$ Paula Preston-Hurlburt, ${ }^{1}$ Mark Mamula, ${ }^{2}$ \\ James C. Reed, ${ }^{1}$ Sindhu Mohandas, ${ }^{3}$ Betsy C. Herold, ${ }^{3}$ Richard Torres, ${ }^{4}$ Silvio M. Vieira, \\ Bentley Lim, ${ }^{5}$ Jose D. Herazo-Maya, ${ }^{2}$ Martin Kriegel, ${ }^{1,2}$ Andrew L. Goodman, ${ }^{5}$ Chris Cotsapas, ${ }^{6}$ \\ and Kevan C. Herold ${ }^{1,2}$ \\ 'Department of Immunobiology, ${ }^{2}$ Department of Internal Medicine, ${ }^{3}$ Department of Pediatrics, Albert Einstein College \\ of Medicine, New York, New York, USA. ${ }^{4}$ Department of Laboratory Medicine, Molecular Biophysics and Biochemistry, \\ ${ }^{5}$ Department of Microbial Pathogenesis and Microbial Sciences Institute, and ${ }^{6}$ Department of Neurology, Yale University \\ School of Medicine, New Haven, Connecticut, USA.
}

The microbiome affects development and activity of the immune system, and may modulate immune therapies, but there is little direct information about this control in vivo. We studied how the microbiome affects regulation of human immune cells in humanized mice. When humanized mice were treated with a cocktail of 4 antibiotics, there was an increase in the frequency of effector T cells in the gut wall, circulating levels of IFN- $\gamma$, and appearance of anti-nuclear antibodies. Teplizumab, a non-FcR-binding anti-CD3 $\varepsilon$ antibody, no longer delayed xenograft rejection. An increase in CD8 ${ }^{+}$central memory cells and IL-10, markers of efficacy of teplizumab, were not induced. IL-10 levels were only decreased when the mice were treated with all 4 but not individual antibiotics. Antibiotic treatment affected CD11b+CD11c cells, which produced less IL-10 and IL-27, and showed increased expression of CD86 and activation of T cells when cocultured with T cells and teplizumab. Soluble products in the pellets appeared to be responsible for the reduced IL-27 expression in DCs. Similar changes in IL-10 induction were seen when human peripheral blood mononuclear cells were cultured with human stool samples. We conclude that changes in the microbiome may impact the efficacy of immunosuppressive medications by altering immune regulatory pathways.

Authorship note: E. Gülden and N.K. Vudattu are co-first authors.

Conflict of interest: The authors have declared that no conflict of interest exists.

Submitted: November 10, 2016 Accepted: September 27, 2017 Published: November 2, 2017

\section{Reference information:}

JCI Insight. 2017;2(21):e91709. https://doi.org/10.1172/jici. insight.91709.

\section{Introduction}

Exposure to microbiota is required for the development of the immune system, immunologic tolerance, and effector function (1-3). Microbiota that lack the resilience and diversity needed to maintain this balance, possibly as a consequence of antimicrobial exposure, are postulated to contribute to the rise in autoimmune diseases, such as inflammatory bowel disease, in Western countries $(4,5)$. A prominent human symbiont, Bacteroides fragilis protects animals from colitis induced by Helicobacter hepaticus by producing polysaccharide A (PSA), which mediates the conversion of $\mathrm{CD}^{+} \mathrm{T}$ cells into Foxp3 ${ }^{+}$Tregs (6-8). Ingested antigens have been shown to induce tolerance. Peripheral Tregs that express ROR $\gamma \mathrm{t}$ and produce IL-10 have been found to be induced in the small intestine by dietary antigens (9).

In addition, immune effector cells may be activated by disturbances in the intestinal microbiota. Gastrointestinal infections can directly affect tolerance to commensals and activate microbiota-specific $\mathrm{T}$ cells that differentiate into inflammatory effector cells (10). Observational studies suggest that restricted diversity of the microbiota may be related to the progression of type 1 diabetes in those at risk (11-13). The composition of the microbiota may also modify responses to biologics and other therapeutics such as the checkpoint inhibitors used for cancer, anti-CTLA-4 or anti-PD-L1 mAbs (14-16). Despite the circumstantial evidence, direct cause/relationships between changes in the microbiota and modulation of human immune responses have not been demonstrated because it is difficult to directly study the effects of changing the microbiota on human immune responses.

Because of the intimate relationship between the microbiota, tolerance, and adaptive immune responses in the gut, we asked whether the microbiota are responsible for maintaining tolerance and its effects on the efficacy of anti-CD3 $\mathrm{mAb}$ in humanized mice. In previous studies in these mice, we described a 
mechanism whereby a non-FcR-binding anti-CD3 mAb (teplizumab) can induce tolerance by generating IL-10-producing cells in the gut, with regulatory function (17-19). Migration of the cells to the gut was needed for these events to occur, and therefore we tested whether the microbiome affected the immune regulatory effects of the $\mathrm{mAb}$. We show that when humanized mice are treated with antibiotics there is breach of tolerance manifested by increased effector $\mathrm{T}$ cells in the lamina propria and development of anti-nuclear antibodies (ANAs), and the ability of teplizumab to prevent xenograft rejection was impaired. Biomarkers associated with efficacy of teplizumab in patients, such as release of IL-10 and expansion of $\mathrm{CD}^{+}$central memory (CD8CM) T cells were seen in humanized mice but not when antibiotics were given (20). The failure to induce regulatory mechanisms could not be attributed to a single microbial species and administration of individual antibiotics from the cocktail of 4 drugs did not induce the same effect. The cells affected directly by the change in microbiome were $\mathrm{CD} 11 \mathrm{~b}^{+} \mathrm{CD} 11 \mathrm{c}^{+}$cells, since direct culture of this subset with pellets from antibiotic-treated mice stimulated lower levels of IL-10 and IL-27 compared with pellets from non-antibiotic-treated mice. Moreover, when $\mathrm{CD} 11 \mathrm{~b}^{+} \mathrm{CD} 11 \mathrm{c}^{+}$cells that had been exposed to pellets from antibiotic-treated mice were added to cultures of $\mathrm{T}$ cells with teplizumab, activation of the $\mathrm{T}$ cells was increased. Likewise, stool samples from antibiotic-treated patients induced less IL-10 compared with patients not treated with antibiotics when cultured with peripheral blood mononuclear cells (PBMCs) from healthy donors. Our studies indicate that there is increased activation of T cells and reduced production of IL-10 when antigen-presenting cells (APCs) are exposed to microbiota from antibiotic-treated individuals and suggest that chronic exposure to the microbiome is needed to maintain tolerance. By modifying immune cells, changes in the microbiome may affect clinical responses to biologics.

\section{Results}

Modification of the microbiome leads to loss of tolerance in humanized mice. Immunodeficient NSG mice, reconstituted with human CD34 ${ }^{+}$stem cells, were treated with a cocktail of 4 antibiotics (neomycin, vancomycin, ampicillin, and metronidazole) at 12 weeks of age after screening. Within 2 weeks, the mice developed a megacecum (Supplemental Figure 1A; supplemental material available online with this article; https:// doi.org/10.1172/jci.insight.91709DS1). There was a reduced number of gut microbial species and changes in the distribution of the microbiota based on analysis of their fecal pellets: Eubacteria were depleted 24 \pm 4.3 -fold (mean $\pm \mathrm{SEM}, n=11$ ) and segmented filamentous bacteria were depleted $34.7 \pm 1.86$-fold (Supplemental Table 1). Tenericutes spp. were the predominant phylum of bacteria remaining in pellets after antibiotic treatment (Figure 1, A and B).

We analyzed immune cells in the tissues to determine the effects of the antibiotic treatment. We did not detect changes in the frequency of subsets of T, B, or NK cells in the spleen even in mice that had received antibiotics for up to 6 weeks (data not shown). There was a modest increase in the number of CD45 cells in the small bowel (Figure 1C; $13,220 \pm 265$ vs. 29,330 $\pm 9,074, P=0.038$ ) but we did not identify lymphocytic infiltrates of graft-versus-host disease in the gut wall (Supplemental Figure 1B). In response to antibiotic treatment there was an increase in the frequency of CD4 $4^{+}$effector T cells $(P=0.040)$ in the gut wall and a decrease in naive cells $(P=0.019)$ with a similar pattern in gut-infiltrating $\mathrm{CD} 8^{+}$cells $(P=$ not significant [ns]) (Figure $1, \mathrm{D}$ and $\mathrm{E})$. In the gut wall, we also observed an increase in $\mathrm{CD} 19^{+}$cells following antibiotic treatment (Figure $1 \mathrm{~F}, P=0.026$ ). The circulating levels of IFN- $\gamma$ were significantly increased in antibiotic-treated mice, consistent with the signs of cell activation among the gut-infiltrating lymphocytes (Figure $1 \mathrm{G}$ ).

Because of the activation of cells in the small bowel, we tested whether the mice had other signs of immune dysregulation. We detected ANAs in 5 of 6 mice treated with antibiotics versus 0 of 4 in humanized mice not treated with antibiotics (Figure 2, $P=0.048$ by $\chi^{2}$ test), indicating that the humanized mice develop signs of systemic immune activation when the microbiota are modified.

Modification of the microbiome impairs tolerance induced by anti-CD3 $\mathrm{mAb}$. We previously showed that teplizumab, an non-FcR-binding anti-CD3 mAb, prevented xenograft skin rejection in humanized mice (17). Because this effect required migration and activation in the gut, we asked whether modification of the microbiome with antibiotics would affect these immunologic actions of teplizumab. We compared the rate of rejection of xenogeneic C56BL/6 skin grafts on mice that were treated or not with antibiotics. Similar to our previous experience, after a single dose of teplizumab the survival of xenogeneic skin grafts was prolonged on humanized mice not treated with antibiotics $(P=0.001$, Figure $3 \mathrm{~A}$ and Supplemental Figure 2). However, in mice treated with antibiotics, teplizumab treatment did not significantly delay graft rejection compared with mice treated with human Ig (hIg) (Figure 3B). 


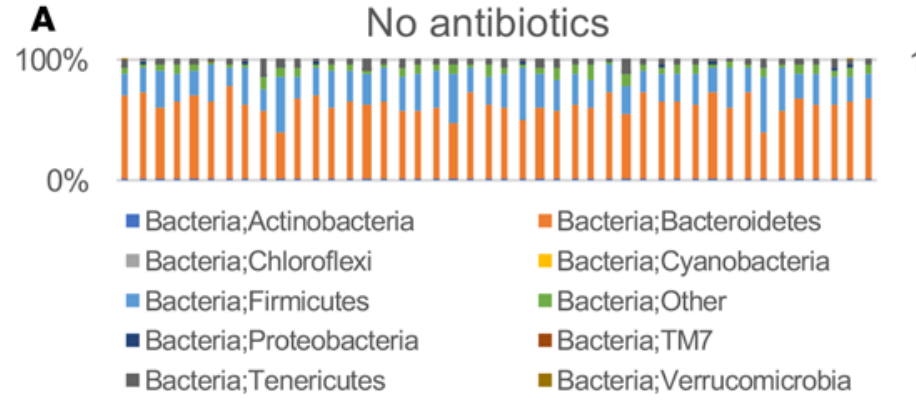

C

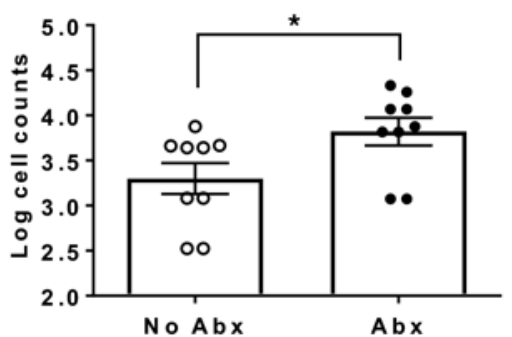

D $\quad \mathrm{CD} 4$

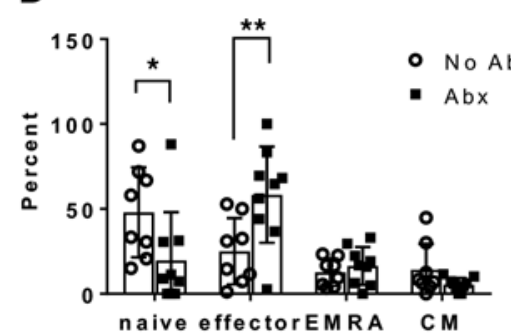

G

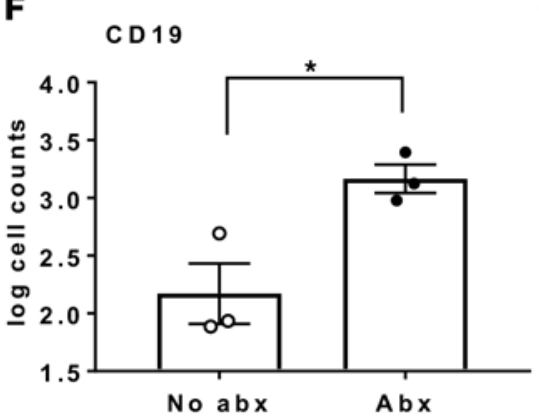

B $100 \%$

$0 \%$ - Bacteria;Actinobacteria - Bacteria;Chloroflexi - Bacteria;Firmicutes - Bacteria;Proteobacteria +Antibiotics
||||||||||||||||||

- Bacteria;Bacteroidetes

= Bacteria;Cyanobacteria

- Bacteria;Other

- Bacteria;TM7

- Bacteria;Verrucomicrobia

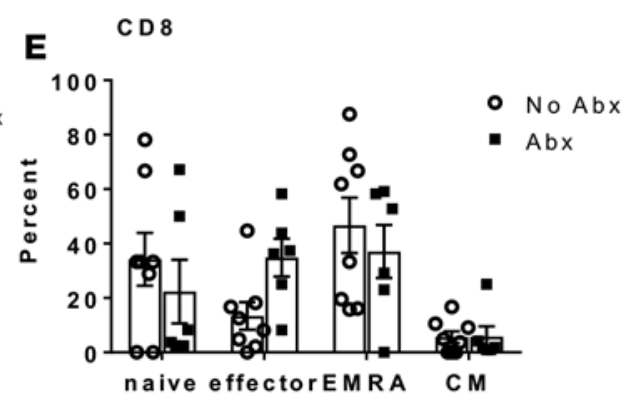

Figure 1. Changes in microbiota and lymphocytes with antibiotics. 165 sequencing was performed on freshly isolated pellets from humanized mice (A) and humanized mice treated with the cocktail of 4 antibiotics for 2 weeks (B). Following antibiotic treatment there was a $24 \pm 4.32$-fold depletion of Eubacteria ( $n=11, P=0.0002)$ and a $34.7 \pm 1.86$-fold depletion of segmented filamentous bacteria $\left(P=4.33 \times 10^{-9}\right)$. The flora was largely restricted to Ternicutes spp. after antibiotic treatment. (C) The number of $C D 2^{+}$cells in the lamina propria of the mice treated with antibiotics (Abx) compared with mice that did not receive antibiotics is shown (each symbol represents an individual mouse, $P=0.0382$, Student's $t$ test). (D) There was a significant interaction between antibiotic treatment and CD4 ${ }^{+} T$ cell subsets in the gut $(P=0.0004,2$-way ANOVA). Naive and effector T cells were decreased and increased, respectively. ${ }^{*} P<0.05,{ }^{*} P<0.01 ; n=8$ and 9 /group. (E) Similar findings were seen within the CD $8{ }^{+} T$ cells but the differences were not statistically significant. (F) The number of $\mathrm{CD}_{19}{ }^{+}$cells in the lamina propria of the mice treated with antibiotics compared with mice that did not receive antibiotics is shown (each symbol represents an individual mouse; $P=0.0261$, Student's $t$ test) (C) The circulating levels of IFN- $\gamma$ were elevated in the antibiotic-treated mice. Each symbol represents an individual mouse that was or was not treated with antibiotics. ${ }^{* *} P=0.008$, Student's $t$ test. CM, central memory T cell; EMRA, effector memory RA T cell.

The differences in the rate of rejection were due to effects of antibiotic treatment, since the rates of rejection in hIg-treated mice were similar whether or not the mice had received antibiotics but different when the mice were treated with teplizumab $(P=0.03)$ (Figure $3, \mathrm{C}$ and $\mathrm{D})$.

Effects of anti-CD3 $m A b$ on adaptive immune cells when the microbiota are modified. The failure of teplizumab to induce tolerance during antibiotic treatment was not because of reduced lymphoid cells in the gut. The cell counts in the lamina propria 18 hours after mice received teplizumab were similar in mice that had been or had not been treated with antibiotics (Figure 4A). Kim et al. found that Tregs induced by dietary antigens expressed ROR $\gamma \mathrm{t}(9)$. We analyzed ROR $\gamma t$ expression by reverse transcription PCR (RT-PCR) in the gut wall and found that the expression was reduced in the mice treated with the antibiotic cocktail (Figure 4B, $P<0.05$ ). We determined the frequency of Tregs in the gut and spleen by staining for $\mathrm{CD} 4^{+} \mathrm{CD} 25^{+} \mathrm{CD} 127^{10} \mathrm{Foxp} 3^{+}$cells but the frequency of these Tregs was variable and we did not identify significant differences in these cells in the gut wall or peripheral sites in mice that had or had not received antibiotics before or after teplizumab treatment (data not shown). 


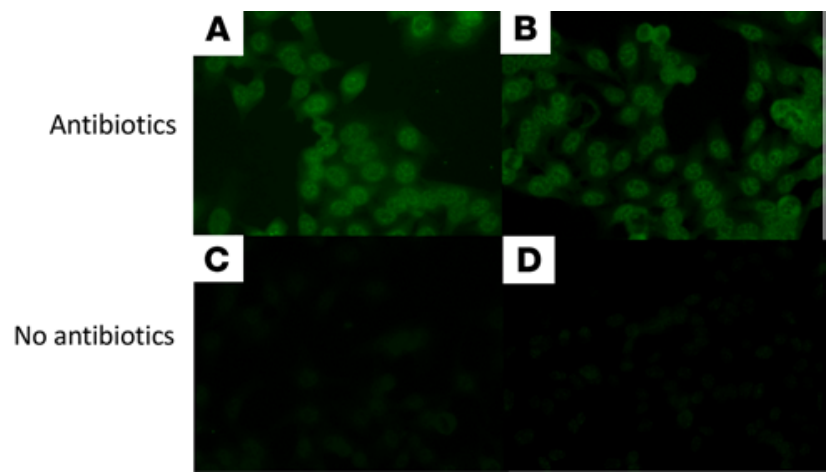

Figure 2. Antinuclear antibodies (ANAs) in humanized mice treated with antibiotics. After 2 weeks of treatment with antibiotics, serum was taken from humanized mice and ANAs were measured using HEp-2 slides. Representative staining is shown for serum from mice treated (A and $\mathbf{B}$ ) or untreated (C and $\mathbf{D}$ ) with antibiotics. Original magnification, $\times 200$. ANAs were detected in 5 of $6(83 \%)$ of the humanized mice that were treated with antibiotics but not $(0$ of 4$)$ in the untreated mice. ${ }^{*} P=$ $0.048, \chi^{2}$ test.

We analyzed T cell subpopulations following teplizumab treatment in the spleen and mesenteric lymph nodes after teplizumab treatment. The mean fluorescence index (MFI) of CD127, which is reduced with $\mathrm{T}$ cell activation (21), was reduced in cells from the mesenteric lymph nodes in mice that were treated with antibiotics even without teplizumab treatment (Figure 5A, $P=0.016$ ) and unlike control mice, did not decrease further with the $\mathrm{mAb}(P=0.02)$.

In patients with type 1 diabetes treated with teplizumab, an increase in CD8CM T cells is a biomarker of responses to the drug $(20,22)$. CD8CM T cells were increased in the mesenteric lymph nodes of the humanized mice treated with teplizumab (Figure 5B, $P=0.049$ ) but not when they received antibiotics (Supplemental Figure 3, $P=0.03$ ). When splenic T cells were activated with PMA/ionomycin, there was a reduced frequency of IL-2-expressing $\mathrm{CD} 4^{+}$and $\mathrm{CD} 8^{+}$cells in mice treated with teplizumab but not when antibiotics were given (Figure $5, C$ and $\mathrm{D}, P=0.037$ and 0.015 , respectively). In addition, the levels of p-STAT5 were increased after culture with IL-2 in mice treated with teplizumab and antibiotics, particularly among the $\mathrm{CD} 8^{+} \mathrm{T}$ cells (Figure 5 , E and F, $P=0.02$ ).

CCR4 expression is associated with migration to the skin and our previous studies have suggested that teplizumab may induce $\mathrm{CCR} 4^{+} \mathrm{CD} 4^{+} \mathrm{T}$ cells with regulatory features such as IL-10 (19). We therefore analyzed the effects of antibiotic treatment on $\mathrm{CCR} 4^{+} \mathrm{CD} 4^{+}$and $\mathrm{CCR} 4^{+} \mathrm{CD} 8^{+} \mathrm{T}$ cells in the gut wall and spleen (Figure 6). In mice treated with antibiotics and teplizumab, the frequency of $\mathrm{CCR} 4^{+} \mathrm{CD} 4^{+} \mathrm{T}$ cells was reduced in the spleen $(P=0.004)$ and $\mathrm{CCR} 4^{+} \mathrm{CD} 8^{+} \mathrm{T}$ cells in the gut and spleen $(P=0.027$ and 0.03 , respectively) compared with mice that had not been treated with antibiotics.

Antibiotic treatment modifies cytokine responses. IL-10 is an important mediator of tolerance in the gut after treatment with anti-CD3 mAb $(17,19,23,24)$. The levels of IL-10 in the serum of the humanized mice were increased following teplizumab treatment $(P<0.001)$ but not when the mice were pretreated with antibiotics (Figure 7A). To determine whether treatment with a single antibiotic was responsible for this response to teplizumab, we measured the levels of IL-10 in the serum after mice were treated with teplizumab and with individual antibiotics or the pool of 4 antibiotics. Cladograms showing 16S sequencing are shown in Supplemental Figure 4 and the fold changes in Eubacteria and segmented filamentous bacteria are shown in Supplemental Table 1. The levels of IL-10 were lower in the mice that received anti-CD3 $\mathrm{mAb}$ and the combination of all 4 antibiotics compared with mice treated with teplizumab without antibiotics (Figure 7B, $P=0.034$ ), but the levels of IL-10 were not reduced when any of the single antibiotics were given with teplizumab.

Modification of dendritic cells by the microbiome. To determine whether qualitative differences in the pellets could account for the changes we observed in cytokine release and to identify which cells were responsible for the changes, we cultured splenocytes from humanized mice, not treated with antibiotics or teplizumab, with inactivated pellets from mice that had or had not received antibiotics. Equal numbers of bacteria were added to the cultures. Compared with pellets from control (no antibiotics) mice, the pellets from antibiotictreated mice stimulated less IL-10 release by splenocytes $(P=0.04)$, but there were no significant differences in the levels of IFN- $\gamma$ or TNF (Figure 8, A-C).

To have sufficient numbers of cells for this study, we used $\mathrm{CD} 4^{+}$and $\mathrm{CD} 8^{+} \mathrm{T}$ cells, B cells, and dendritic cells (DCs) sorted from PBMCs from healthy donors to determine which cells were affected by the modified microbiome, and cultured them with the pellets from untreated and antibiotic-treated mice (Figure 8D). The DCs, but not T or B cells, released high levels of IL-10 after culture with the 
A

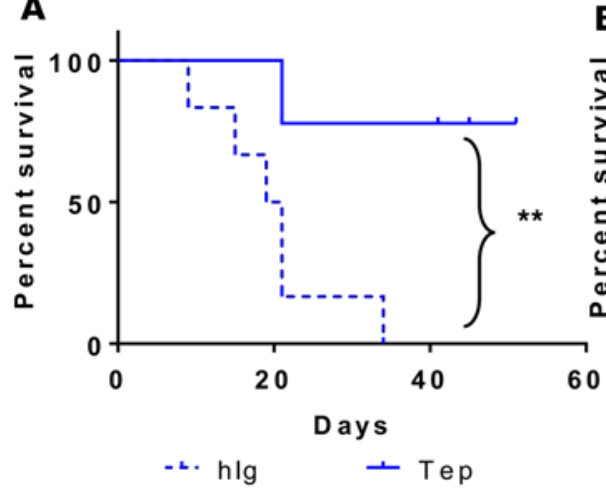

C

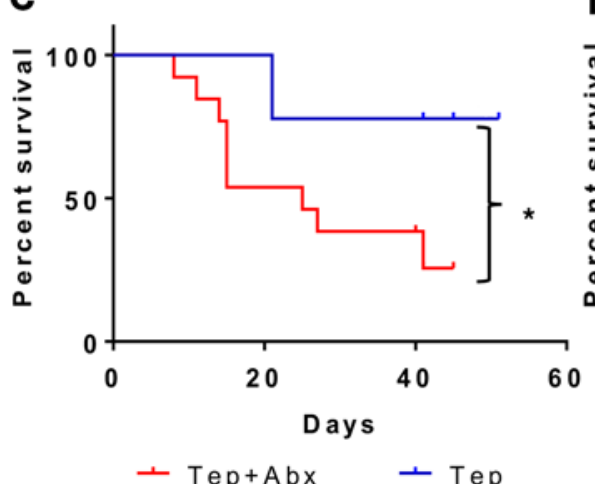

B

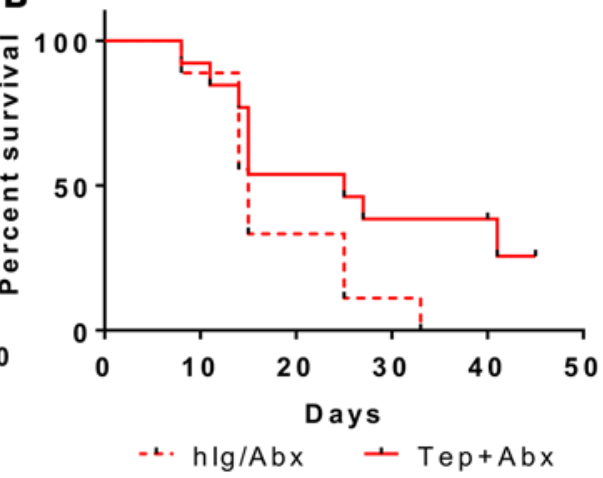

D

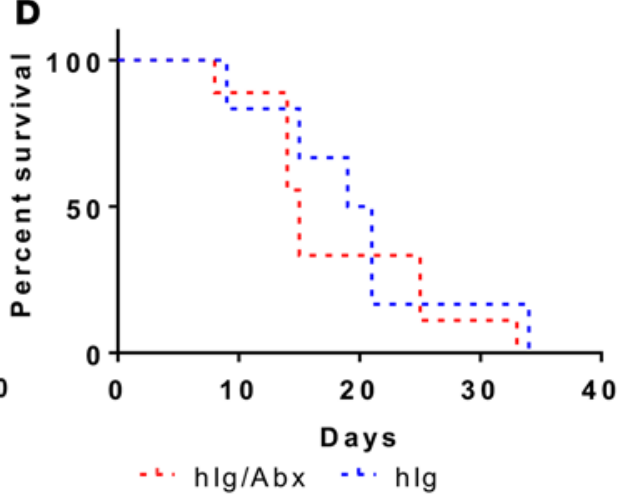

Figure 3. Effects of antibiotics on activity of teplizumab. The rate of skin graft rejection was compared in humanized mice that were treated with teplizumab (Tep) (solid line, $n$ = 9) or hlg (dotted line, $n=6$ ) without antibiotics (Abx) (A) or with antibiotics (B) (hlg, dotted line, $n=9$; teplizumab, solid line, $n=13, P=\mathrm{ns}$ ). (C) There was a significant difference in the rate of graft rejection following teplizumab treatment with (red) or without (blue) antibiotics. (D) The rates of rejection were similar in the hlg-treated mice whether or not (red or blue, respectively) they received antibiotics. ${ }^{*} P$ $=0.026,{ }^{*} P=0.001$, log-rank Mantel-Cox test.

pellets $(P<0.0001, P=0.0008)$, but less when the pellets were from antibiotic-treated mice $(P=$ 0.03). The effects of the antibiotic-treated pellets on $\mathrm{CD} 11 \mathrm{~b}^{+} \mathrm{CD} 11 \mathrm{c}^{+}$cells were restricted to IL-10 $(P<0.0001)$ because release of IL-6, TNF, IL-1 $\beta$, and IL-8 was not significantly different (Figure 8 , E-I). We then tested whether there were similar effects of antibiotic treatment on responses to human microbiota. We cultured human PBMCs with heat-inactivated stool samples from 18 subjects receiving antibiotics and 11 subjects who were not receiving antibiotics and had not received them within the previous 3 months (Supplemental Table 2). We added an equivalent number of organisms $\left(2.2 \times 10^{6}\right)$ to the cultures for 5 days. We tested each fecal sample against PBMCs pooled from 4 different individuals to minimize variability of the responder cells and compared cytokine levels in the supernatants. There was significantly reduced production of IL-10 by PBMCs cultured with the bacteria from stool samples from antibiotic-treated compared with control subjects (Figure 8J, $P=0.0017$ ).

To determine whether the changes in DCs affected the activation of $\mathrm{T}$ cells, we isolated $\mathrm{CD} 11 \mathrm{~b}^{+} \mathrm{CD} 11 \mathrm{c}^{+}$cells from the PBMCs of healthy cell donors and cultured them overnight with equal numbers of bacteria from inactivated pellets from mice that had or had not received antibiotics. There was increased expression of CD86 on the $\mathrm{CD} 11 \mathrm{~b}^{+} \mathrm{CD} 11 \mathrm{c}^{+}$cells from the cultures with antibiotic-treated pellets (Figure 9A, $P=0.02$ ). We then added autologous $\mathrm{T}$ cells with teplizumab and measured activation of the T cells by expression of CD69 after overnight culture. When precultured with inactivated fecal bacteria from pellets from mice treated with antibiotics, there was increased expression of CD69 on $\mathrm{CD}^{+}(P=0.02)$ and $\mathrm{CD}^{+}(P=0.04) \mathrm{T}$ cells compared with pellets from mice that had not been treated (Figure 9, B and C). Thus, pellets from the antibiotic-treated mice enhance the activation of $\mathrm{T}$ cells that are cocultured with $\mathrm{CD} 11 \mathrm{~b}^{+} \mathrm{CD} 11 \mathrm{c}^{+}$cells and teplizumab.

IL-27 has been shown to play an important role in IL-10 expression and development of Tregs that specialize to control Th1 cell-mediated immunity $(25,26)$. Several factors, including IL-10, TGF- $\beta$, and vitamin D have been shown to modulate DCs and induce tolerogenic DCs, which produce IL-27 (27). To understand the basis of changes in the DC population, we analyzed the expression of IL-27 by $\mathrm{CD} 11 \mathrm{~b}^{+} \mathrm{CD} 11 \mathrm{c}^{+}$cells that had been exposed to pellets from non-antibiotic-treated or antibiotictreated mice $(28,29)$. Intracellular expression was similar in $\mathrm{CD}_{11} \mathrm{~b}^{+}$macrophages but was reduced in $\mathrm{CD} 11 \mathrm{~b}^{+} \mathrm{CD} 11 \mathrm{c}^{+} \mathrm{DCs}$ that were cultured with pellets from antibiotic-treated compared with pellets from 
A

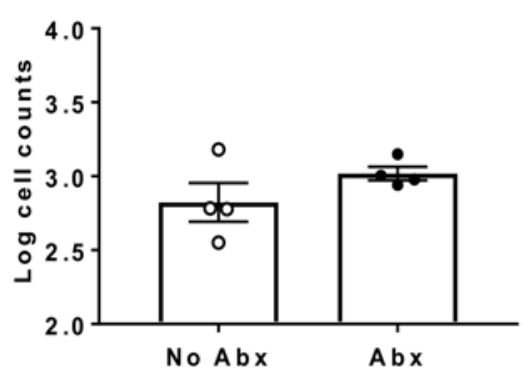

B

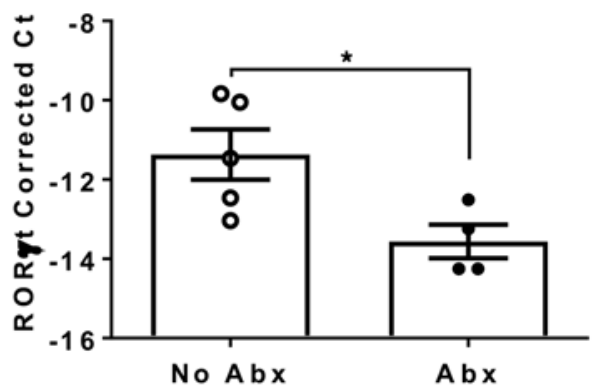

Figure 4. Effects of antibiotic treatment on cells in the gut wall after teplizumab treatment. (A) The number of CD2 $T$ cells in the lamina propria was determined 18 hours after treatment with teplizumab in mice that had or had not received antibiotics (Abx). (B) Gene expression of ROR $\gamma t$ was analyzed by RT-PCR in gut cells 18 hours after treatment with anti-CD3 mAb in mice that had or had not received antibiotics. There was a significant reduction in the expression of ROR $\gamma \mathrm{c}$ in the antibiotic-treated mice. ${ }^{*} P=0.035$, Student's $t$ test; each symbol represents an individual mouse.

mice not treated with antibiotics (Figure 10A, $P=0.02$ ). To determine whether soluble or insoluble products were involved, we suspended the pellets in PBS and cultured the CD11 $\mathrm{b}^{+} \mathrm{CD} 11 \mathrm{c}^{+}$cells with the supernatants or insoluble material from these suspensions. There was significant reduction in the IL-27 expression in the $\mathrm{CD} 11 \mathrm{~b}^{+} \mathrm{CD} 11 \mathrm{c}^{+}$cells that were cultured with the supernatants from the pellets from the antibiotic-treated versus non-antibiotic-treated mice (Figure 10B, $P=0.012$ ).

\section{Discussion}

Our studies show that antibiotic treatment can modify regulation of the human adaptive immune system and break immunologic tolerance. When humanized mice were treated with antibiotics, there was reduced diversity and total bacterial load of the microbiota. Three observations show how the change in the microbiome affected immune tolerance. There was spontaneous development of autoantibodies (ANAs), and increased levels of IFN- $\gamma$ in the circulation with activation of cells in the gut wall. Previously we found that teplizumab treatment prevented xenogeneic skin graft rejection in humanized mice by inducing $\mathrm{T}$ cells with regulatory function $(17,30)$, which we now show is modified by antibiotic treatment. The tolerogenic response to teplizumab requires migration to the gut and activation of cells at that location but the antibiotic treatment did not affect cell migration. Biomarkers of teplizumab action such as increased IL-10 in the serum and expansion of CD8CM T cells were not detected when antibiotics were given. The cocktail of 4 but not individual antibiotics affected IL-10 levels, suggesting that a single organism is not likely to be involved. $\mathrm{CD} 11 \mathrm{~b}^{+} \mathrm{CD} 11 \mathrm{c}^{+} \mathrm{DCs}$ appear to account for these effects since antibiotic treatment caused reduced IL-10 and IL-27 production by these cells when they were cultured with pellets from the antibiotictreated mice. Soluble products produced by the microbiome account for the differences in IL-27 production by the DCs when mice are treated with antibiotics. Finally, we found a similar effect on IL-10 production by human PBMCs that were cultured with the microbiota from subjects that had or had not been treated with antibiotics, suggesting that these observations have relevance for immune responses in patients. Our studies suggest that modification of the microbiome by antibiotics can break tolerance through actions on DCs in the host. They suggest a mechanism whereby antibiotic use may foster autoimmunity and account for differences in individual responses to tolerogenic therapies.

The activated $\mathrm{T}$ cells that were found in the mice treated with antibiotics may not be susceptible to the action of teplizumab or cells induced by teplizumab. In clinical studies, we have shown expansion of CD8CM $\mathrm{T}$ cells that show transcriptional profiles of tolerance such as decreased expression of the IL-7 receptor and increased expression of IL-10 (20). Cell tracing studies showed that in vitro the major source of these cells is the naive $\mathrm{CD}^{+} \mathrm{T}$ cell pool, which was decreased in the antibiotic-treated mice and therefore may account for the failure of the biologic. Our clinical studies also showed that teplizumab induced CCR $4^{+} \mathrm{IL}-10^{+} \mathrm{CD} 4^{+}$ $\mathrm{T}$ cells in the peripheral blood, which several investigators have shown have regulatory function $(19,31-34)$. $\mathrm{CCR}^{+} \mathrm{CD} 4^{+}$and $\mathrm{CCR} 4^{+} \mathrm{CD} 8^{+}$cells were reduced in frequency by antibiotic treatment, suggesting that the microbiome was needed for their induction. Therefore, in addition to activation of $\mathrm{T}$ cells which may render them resistant to regulation, the antibiotic treatment also affected cells that can mediate immune regulation. 
A

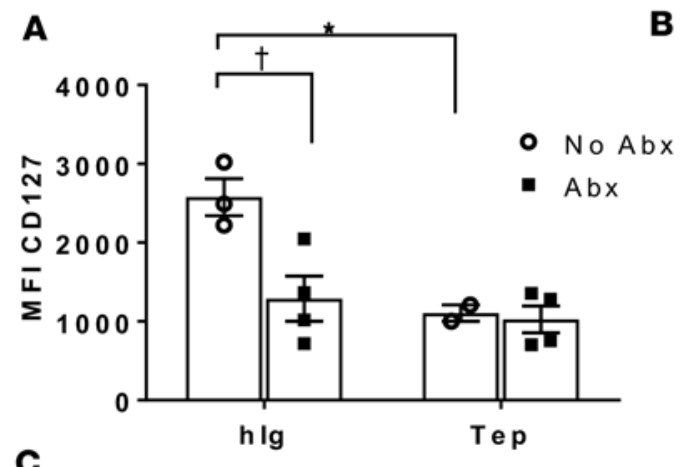

C

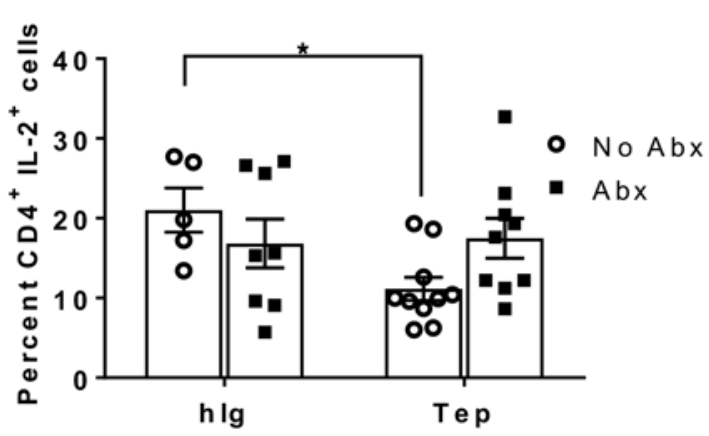

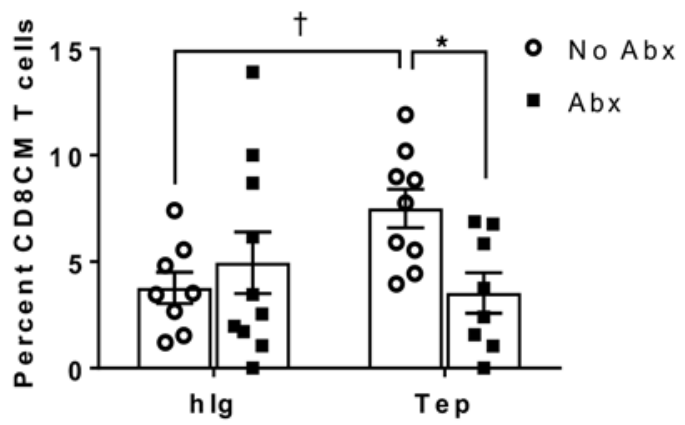

D

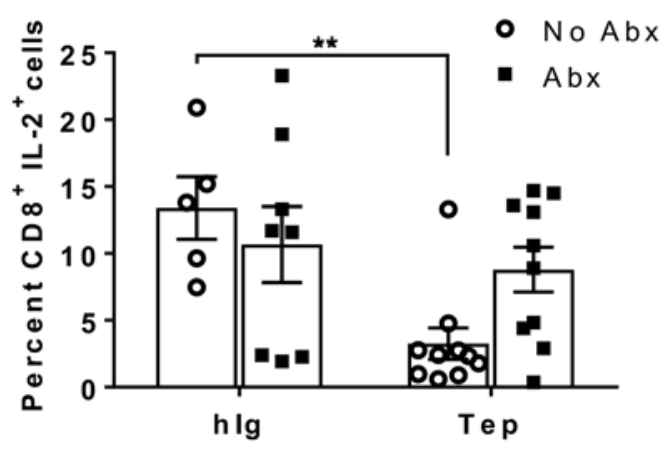

F Tep

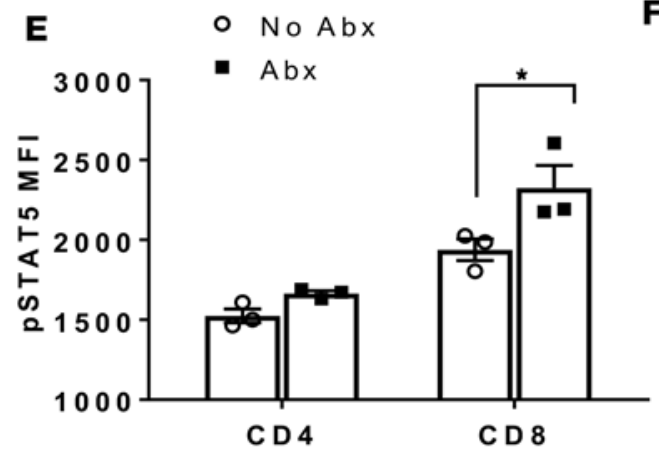

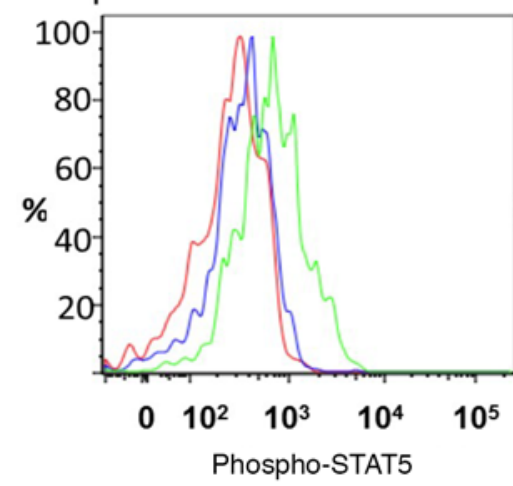

Tep+Antibiotics

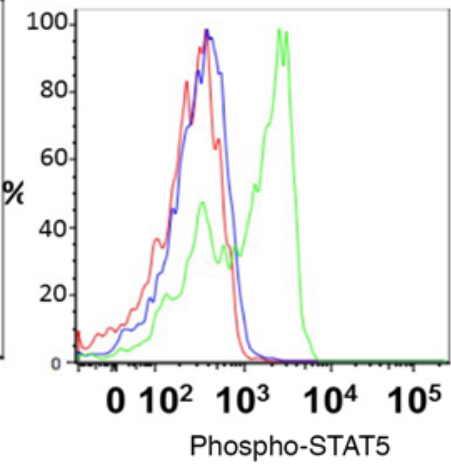

Figure 5. Effects of antibiotic treatment on peripheral cells. (A) The expression of CD127 was measured on CD2 ${ }^{+}$cells in the mesenteric lymph nodes (MLNs) in mice treated with hlg or teplizumab in the presence or absence of antibiotics (Abx). In hlg- and antibiotic-treated mice, CD127 expression was reduced compared with mice that had not been treated with antibiotics $\left({ }^{\dagger} P=0.016\right)$. The MFI was significantly reduced after teplizumab in mice that had not received antibiotics ( $\left.{ }^{*} P=0.02\right)$ but not in the mice treated with antibiotics ( $n=3-4$ mice/group). (B) There was an interactive effect of antibiotic treatment on the frequency of CD8CM in the mesenteric lymph nodes after teplizumab treatment $\left.{ }^{*} P=0.035\right)$. The frequency increased in mice that received teplizumab without antibiotics $\left({ }^{\dagger} P=0.049\right)$ but not in mice that received antibiotics $(n=3-4$ mice/group), and was higher than in mice treated with teplizumab and antibiotics $(P=0.035)$. ( $($ and $\mathbf{D})$ The frequency of $\mathrm{IL}-2$-producing $\mathrm{CD} 4^{+}$and $\mathrm{CD} 8^{+} \mathrm{T}$ cells in the spleen was analyzed by flow cytometry following stimulation with PMA/ionomycin for 6 hours, 18 hours following treatment with teplizumab or hlg $(n=10$ per group). There was a significant effect of antibiotic treatment on the frequency of IL-2+ CD4 ${ }^{+}$and $C D 88^{+}$T cells. ${ }^{*} P=0.03,{ }^{* *} P=0.006,2$-way ANOVA. (E) p-STAT5 was increased in the CD8 ${ }^{+}$ splenocytes from mice treated with antibiotics and anti-CD3 mAb compared with mice that received anti-CD3 mAb without antibiotics $\left({ }^{*} P=0.02,2\right.$-way ANOVA; each symbol represents 1 mouse). (F) Representative histograms from a mouse treated with teplizumab alone or teplizumab plus antibiotics are shown (red $=$ isotype control, blue $=\mathrm{p}$-STAT5 staining without IL-2, green $=\mathrm{p}-\mathrm{STAT5}$ staining with IL-2). Statistical significance in all panels was determined by multiple comparison from 2-way ANOVA.

We identified $\mathrm{CD} 11 \mathrm{~b}^{+} \mathrm{CD} 11 \mathrm{c}^{+}$cells as the sensor cells for the microbiome. Rather than a single organism, our findings with individual antibiotics and cultures with supernatants and insoluble material from the pellets suspended in PBS suggest that soluble products account for the effects on IL-27 production. Further studies and correlations between the presence or absence of bacteria and loss of IL-10 in the humanized mice will be needed to determine whether specific families of organisms are responsible. One possibility is that the metabolites derived from commensal bacteria such as short-chain fatty acids (SCFAs), which include butyrate, propionate, and acetate that have the potential to change the epigenome of host cells that control development and function 

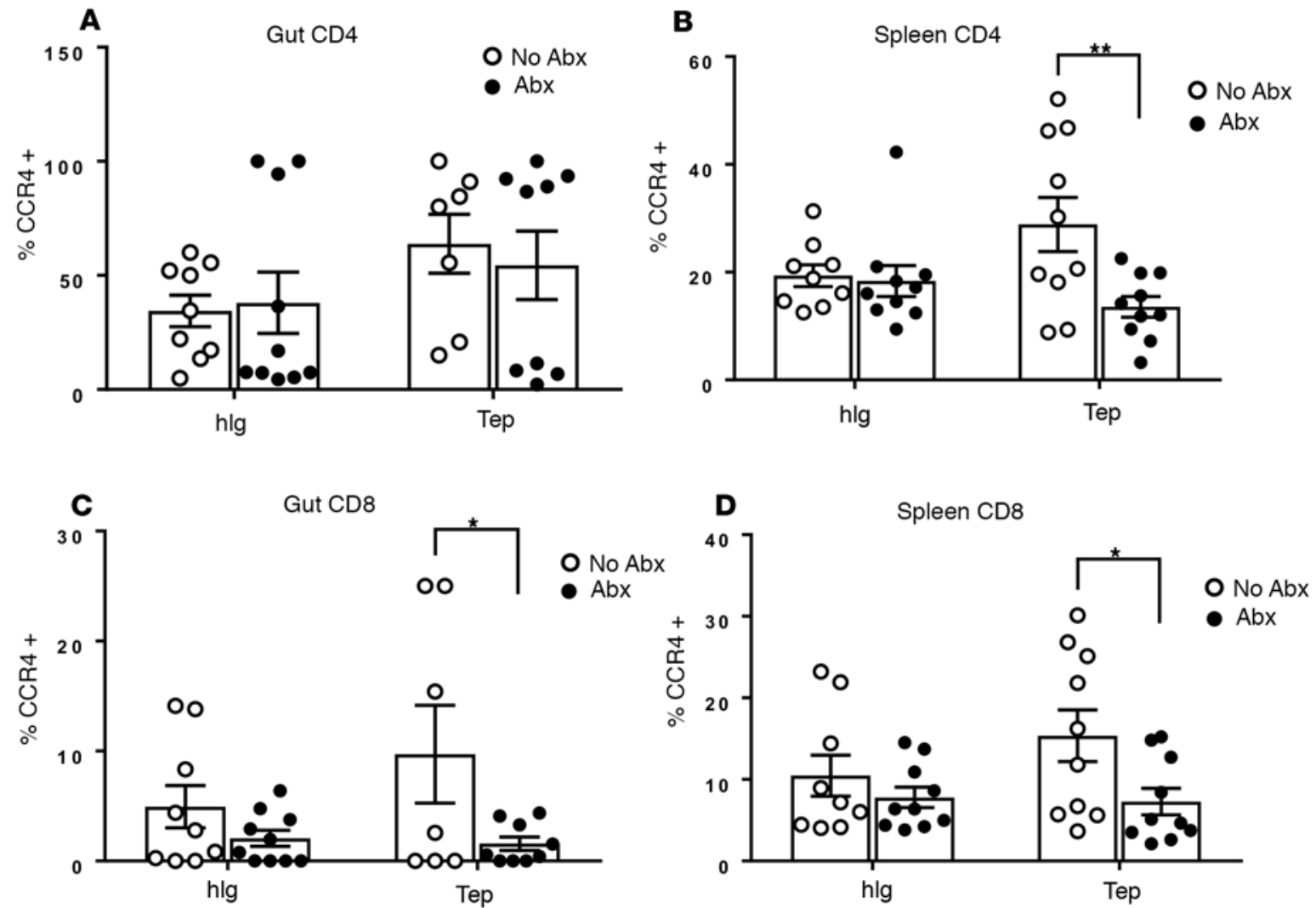

Figure 6. CCR4 ${ }^{+}$CD4 ${ }^{+}$and CCR4+CD8+ $\mathbf{T}$ cells in the gut wall and spleen. The expression of CCR4 on CD4 ${ }^{+}$cells in the gut (A) and spleen (B) and on CD8 ${ }^{+}$cells in the gut (C) and spleen (D) was measured by flow cytometry in cells that were harvested 18 hours after mice that had (black bars) or had not (open bars) been treated with hlg or teplizumab (Tep). ${ }^{*} P<0.05$, ${ }^{* *} P<0.01$ by 2 -way ANOVA ( $n=7-10$ mice/group). Abx, antibiotics.

of Tregs in the gut, are no longer produced $(35,36)$. Interestingly, in mice, Bifidobacterium breve was shown to induce IL-10- and IL-27-producing Tr1 cells in the colon via a TLR2/MyD88 pathway, consistent with our findings of activation with the pellets from the antibiotic-treated mice (37). Eubacteria can produce butyrate, which induces gene expression changes in DCs leading to suppression of IL-12, IL-6, and expression of RELB, which contributes to Treg differentiation $(38,39)$. Butyrate has also been linked to IL-10 production through the activation of the niacin receptor Gpr109a (40). Although further studies to directly demonstrate an association between these organisms together or individually and IL-10 production are needed, these observations suggest that the normal microbiome maintains tolerance of human cells by the balance of metabolites that are produced.

An alternative explanation may also account for our findings about the activation properties of the $\mathrm{CD} 11 \mathrm{~b}^{+} \mathrm{CD} 11 \mathrm{c}^{+}$cells and the microbiome. Rather than the absence of an inducer of immune regulation the absence of an immune activator may be involved. Watson and Kim described the phenomenon of "endotoxin tolerance" in which stimulation of immune cells with LPS induces a refractory state to a repeated immune challenge (41). Vatanen et al. found that LPS from different strains of commensals differed in their ability to induce this state and may account for susceptibility or resistance to autoimmune diabetes in genetically similar individuals from Russia and Finland (13). The failure to induce a state like endotoxin tolerance is consistent with the enhanced $\mathrm{T}$ cell activation when CD11c cells were cultured with pellets from the antibiotic-treated mice. In line with this notion is also the fact that IL-10, serum levels of which are higher in non-antibiotic-treated mice and which is more highly expressed by $C D 11 b^{+} C D 11 c^{+}$ cells in response to fecal samples of untreated mice, is known to block induction of TLR4 by LPS (42). Regardless of the cause, the changes in the microbiome lead to DC activation and increase the activation of T cells when cultured with teplizumab. Although teplizumab is non-FcR binding, in previous studies we have shown that the effects of the mAb on T cells requires the presence of APCs (18).

There are limitations to our findings. The antibiotic treatment affects the microbiota at other locations as well as the gut, and these effects, as well as direct effects of the drugs on immune cells, may be involved. Studies from other investigators suggest this is not the case, since immunologic effects that were seen in antibiotic- 

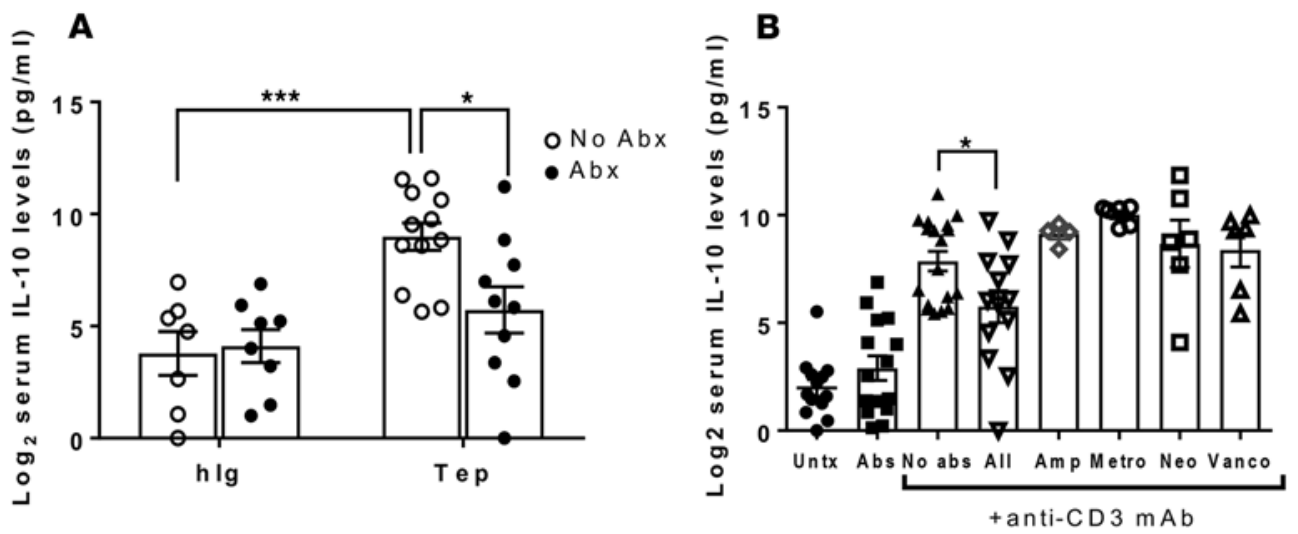

Figure 7. Effects of antibiotic treatment on IL-10 levels. (A) Serum was harvested from the humanized mice with skin grafts that had $(n=12)$ or had not $(n=7)$ been treated with antibiotics (Abx) 18 hours after teplizumab (Tep) or control Ig (hlg) treatment. The levels of IL-10 were increased after teplizumab in the mice but not when they were treated with antibiotics. There was significantly less IL-10 after teplizumab in the mice that had versus had not received antibiotics. ${ }^{*} P<0.03,{ }^{* *} P=0.0009,2$-way ANOVA. (B) The serum levels of IL-10 were compared in mice treated with all 4 or the individual antibiotics 18 hours after teplizumab ( $n=4-18 /$ group). The levels of IL-10 were reduced in the mice treated with all 4 antibiotics (All) but not in the mice treated with the individual antibiotics compared with the mice that did not receive antibiotics. ${ }^{*} P=0.03$, ANOVA with Dunnett's multiple comparison test. Amp, ampicillin; Metro, metronidazole; Neo, neomycin; Vanco, vancomycin; Untx, untreated.

treated mice were similar to gnotobiotic-treated mice (14-16), and our studies with pellets from the antibiotictreated mice and human samples suggest that the direct effects of the antibiotics on the gut flora rather than the host are responsible. Although we did not detect effects of antibiotic treatment on gut migration of $\mathrm{T}$ cells, we cannot exclude the possibility that failure to induce tolerance was associated with enhanced migration of effector cells to the skin grafts, which may be modulated when the microbiota are present. Because this humanized mouse model system does not have a thymus, studies of the function of Tregs are not optimal in this model. In addition, some tolerance mechanisms that may be important even when the microbiome is altered differ in the humanized mice from those in normal humans. Central tolerance and peripheral ligands such as PD-L1 and other immune inhibitory receptors may not cross react between mouse and human cells.

In summary, our studies show that the microbiome is needed to maintain tolerance by human immune cells. Our findings highlight the critical role of the microbiota in this setting since teplizumab restored tolerance when autoimmunity was induced by other means (i.e., anti-CTLA-4 mAb) (30). These observations may have broad application to understanding the basis for autoimmune diseases and the increased frequency of these ailments that have been associated with the avoidance of infections and use of antibiotics in Western countries (43). In addition, our studies suggest a mechanism that could account for individual differences in responses to biologics. Further studies from clinical trials may help to determine whether consideration of the microbiota can be used to personalize treatments for immune therapies.

\section{Methods}

Humanized mice. NOD/SCID IL-2 $\gamma^{-/-}$(NSG) mice were purchased from The Jackson Laboratory, and bred and housed in our animal facility under specific pathogen-free conditions. CD $34^{+}$cells were isolated from 11 different donors and purified with magnetically labeled microbeads conjugated to anti-human CD34. The cells $\left(1 \times 10^{5}\right)$ were injected intrahepatically into irradiated (1 Gy) NSG pups within 48 hours of birth. The reconstitution of the mice was determined in peripheral blood at 12 weeks of age. Mice that showed at least $50 \%$ of hCD $45^{+}$cells in the peripheral blood were used for studies.

Antibiotic treatment. A cocktail of vancomycin (0.5 g/1), ampicillin (1 g/1), neomycin ( $1 \mathrm{~g} / 1)$, and metronidazole $(1 \mathrm{~g} / 1)$ was added to the drinking water. The antibiotic-containing drinking water was continued until the time of sacrifice.

Fecal microbiota from the mice were collected before and after 2 weeks of antibiotic treatment and frozen at $-80^{\circ} \mathrm{C}$. Fecal pellets were weighed and bacterial DNA was isolated using a PowerSoil DNA isolation kit (Mo Bio). Equivalent amounts of DNA were used for analysis of eubacterial and segmented filamentous bacterial contents by quantitative PCR using the SyberGreen protocol. V4-directed 16S rRNA 

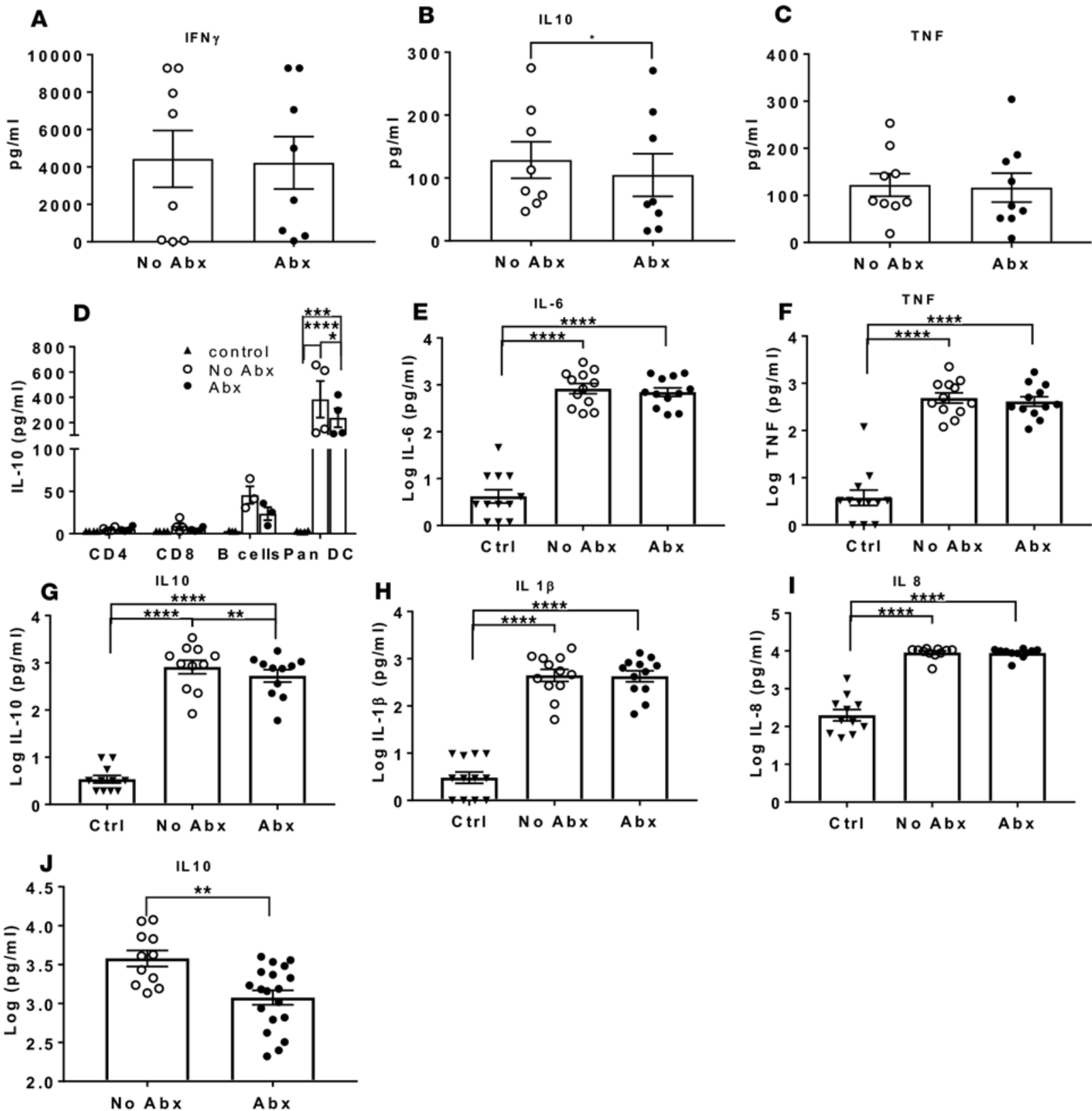

Figure 8. Effects of antibiotic treatment on pellets and cells from humanized mice. (A-C) Splenocytes from mice that had not been treated with antibiotics (Abx) were cultured with pools of pellets from mice that had not or had been treated with antibiotics. The levels of IFN- $\gamma$, IL-10, and TNF were measured in the supernatants after 5 days and compared by paired $t$ test. ${ }^{*} P=0.04, n=8$ cell donors/group. (D) Subsets of human PBMCs were isolated by magnetic beads and cultured with pellets from mice that had or had not been treated with antibiotics $(n=5$ each). There was increased IL-10 release when the DCs were cultured with pellets from both groups of mice but reduced release with pellets from the antibiotic-treated mice. ${ }^{*} P=0.03,{ }^{* * *} P=0.0008$, ${ }^{* * * *} P<$ 0.0001, 2-way ANOVA with paired comparisons. (E-I) DCs were isolated from the PBMCs from healthy donors and cytokine levels were measured after 5 days in culture with a pool of pellets from mice that had or had not been treated with antibiotics. Each symbol represents a DC cell donor. Culture with the pellets increased production of all cytokines $(P<0.0001)$ compared with the cultures without pellets $(C \mathrm{Crl})(P=0.002$ by ANOVA). (J) Human stool samples of antibiotic-treated patients and healthy controls were heat inactivated and cultured with PBMCs that were pooled from 4 healthy cell donors. The level of IL-10 in the supernatant after culture for 3 days is shown. Each symbol represents a stool sample donor. ${ }^{* *} P=0.002,{ }^{* * * *} P<0.0001$ by Student's $t$ test.

gene sequencing was performed as described (44). Briefly, amplicons were generated using dual-barcoded primers (45), pooled, and sequenced using $2 \times 250$ bp chemistry on an Illumina MiSeq instrument. Pairedend reads were assembled and clustered into operational taxonomic units (OTUs) at $97 \%$ identity using an open-reference method. Taxonomy was assigned using the Greengenes gg_13_5_otus reference database (46) and OTUs rarefied to 20,000 sequences per sample and singletons excluded prior to further analysis. 
A

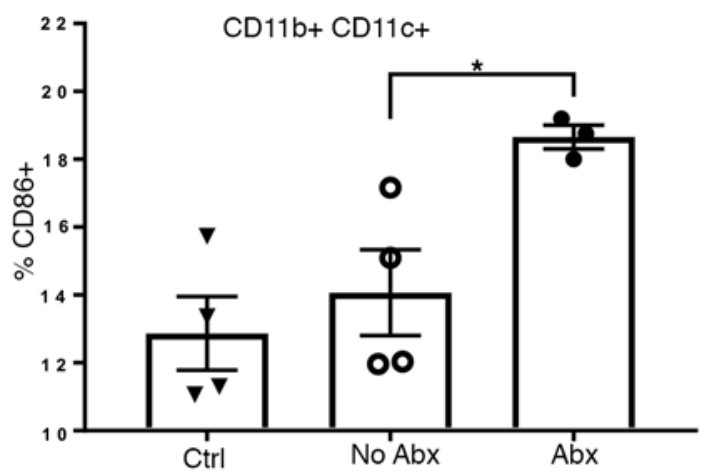

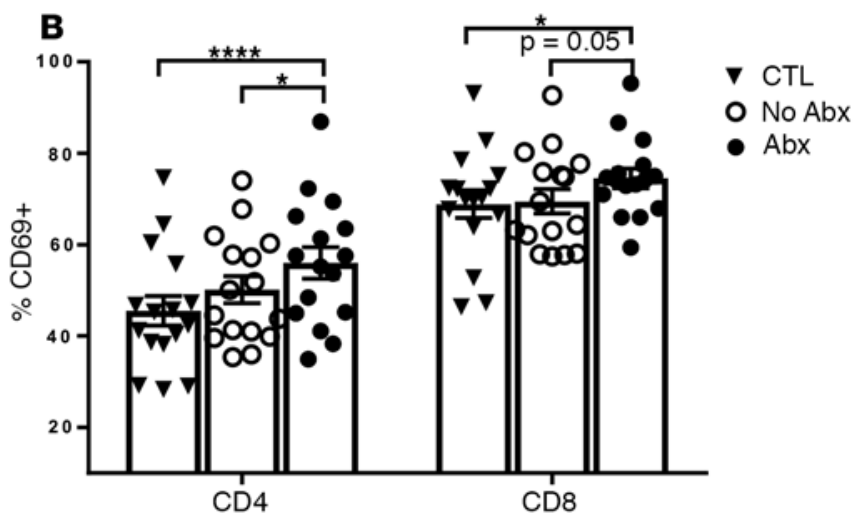

Figure 9. DCs cultured with antibiotic-treated pellets enhance T cell activation with anti-CD3 mAb. CD11 ${ }^{+}$cells were isolated from PBMCs and cultured with pellets as described in the Methods. (A) The expression of CD86 on CD11b ${ }^{+} C D 11 c^{+} D C s$ cultured in pellets from antibiotic-treated mice was increased. ${ }^{*} P=0.02$ by multiple comparison from ANOVA. (B) Autologous T cells were then added to the cultures with teplizumab and the expression of CD69 was measured on the $\mathrm{CD}^{+}$and $\mathrm{CD} 8^{+} \mathrm{T}$ cells after overnight culture. There was increased expression of CD69 on CD8 ${ }^{+} \mathrm{T}$ cells that had been cultured with cells and antibiotic-treated pellets. ${ }^{*} P<0.05,{ }^{* * *} P<0.0001$ by 2-way ANOVA with paired comparisons. Abx, antibiotics.

Skin grafts. Mouse full-thickness skin xenografts from C57B6/J RAG ${ }^{-/-}$mice were transplanted on the dorsum of the recipient humanized mice. The next day either hIg or teplizumab (humanized, non-FcRbinding anti-CD3 mAb, gift of MacroGenics, Inc, Rockville, Maryland, USA) (5 $\mu \mathrm{g})$ was given i.p. Some mice were sacrificed after 18-22 hours, others after 10 days, and the organs and sera were collected. Dressings were removed under anesthesia at day 7 . The skin grafts were monitored daily and the day of rejection was assessed by 2 observers and defined as the day when the graft was no longer present.

ANAs. ANA testing was performed with HEp-2 slides that were incubated with a 1:40 dilution of serum samples collected from both control and experimental mice. The serum was incubated with the slides for 1 hour at room temperature in a humidifying chamber. Excess unbound antibodies were removed by washing for 5 minutes in PBS. The slides were incubated with secondary antibody at a 1:100 dilution in PBS for 1 hour, after which they were washed in PBS for 5 minutes and mounted. Images were rendered using an Olympus BX40 microscope. Without knowledge of the mouse donor, they were scored from +1 to +4 , depending on the intensity of staining for ANA.

Immune cell phenotypes. Cells were isolated from spleen, peripheral blood, and mesenteric lymph node following tissue homogenization. RBCs in peripheral blood and spleen were lysed using ACK lysis buffer (Lonza). The entire small intestine was resected and the cells were isolated by digesting the gut with collagenase for 20 minutes followed by 2 washes with sterile cold PBS. The immune cells were purified on a Ficoll density gradient and counted using BD Trucount beads during flow analysis with a FACSAria (BD Biosciences). $\mathrm{T}$ cell phenotypes were determined by flow cytometry using the following antibodies purchased from Biolegend and BD: CD45 (HI30) APC, anti-mouse CD45 (30-F11) Pacific Blue, CD19 (SJ25C1) PE, CD3 (OKT-3) FITC, CD4 (RPA-T4) APC-H7, CD8 (RPA-T8) PerCP-Cy5.5, CD45RO (UCHL-1), CCR7 (GO43H7) PE, CD45RA (HI100) APC-H7, CD103 (Ber-ACT8), CCR4 (L291H4), and $\mathrm{CD} 2$ (RPA-2.10). T cell memory phenotypes were identified as naive $\left(\mathrm{CD} 45 \mathrm{RO}^{-} \mathrm{CCR} 7^{+}\right)$, central memory $\left(\mathrm{CD} 45 \mathrm{RO}^{+} \mathrm{CCR} 7^{+}\right)$, effectors $\left(\mathrm{CD}^{2} 5 \mathrm{RO}^{+} \mathrm{CCR}^{-}\right)$, and effector memory RA (CD45RO-CCR7-). Tregs were identified with CD2 (RPA-2.10), CD4 (RPA-T4), CD25 (2A3), CD127 (A019D5), and Foxp3 (259D). The phenotypes of $\mathrm{CD} 11 \mathrm{~b}^{+}$and $\mathrm{CD} 11 \mathrm{c}^{+}$cells (identified with ICRF44 and Bu15, respectively) were determined using CD86 (IT2.2) APC. CCR4 (ab1664) antibody was purchased from Abcam. For intracellular cytokine staining, splenocytes or $1 \times 10^{6} \mathrm{PBMCs}$ were stimulated with PMA/ionomycin for 6 hours in the presence of GolgiStop and stained with intracellular antibodies by using a BD Fix/Perm kit.

Stat5 phosphorylation in T cells. Constitutive and IL-2-induced STAT5 phosphorylation was evaluated in $\mathrm{CD}^{+} \mathrm{CD}^{+}$or $\mathrm{CD}^{+}$splenocytes after stimulation with IL-2 in vitro (100 U/well). Splenocytes were starved overnight in a serum-free medium (AIM-V from Invitrogen) followed by incubation with $100 \mathrm{U}$ recombinant human IL-2 per $10^{5}$ cells for 20 minutes at $37^{\circ} \mathrm{C}$. The cells were stained, fixed, and permeabilized as described previously (30) and then stained with anti-p-STAT5 (Y694)-Alexa 488 (BD Biosciences, catalog 612598, clone 47). The relative expression of p-STAT5 (MFI) was determined by flow cytometry. 


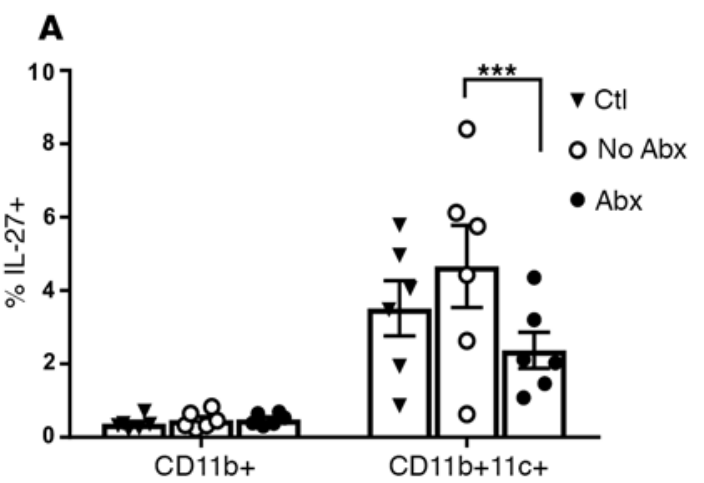

Figure 10. Effects of pellets on IL-27 production. (A) IL-27 expression by intracellular cytokine staining in CD11b+ and $\mathrm{CD}_{11} \mathrm{~b}^{+} \mathrm{CD} 11 \mathrm{C}^{+}$cells isolated from PBMCs cultured with pellets from mice that had or had not been treated with antibiotics (Abx). Ctrl (triangles) represents IL-27 expression in cells that had not been cultured with pellets. There was reduced IL-27 expression in cells exposed to pellets from mice that had been treated with antibiotics. ${ }^{* *} P=0.0007$ by ANOVA with paired comparisons. (B) Pellets were harvested from 6 mice that had not (open circles) or had (closed circles) been treated with antibiotics and dissolved in PBS. The insoluble material or supernatants were cultured with CD11 $\mathrm{b}^{+}$CD11c ${ }^{+}$ cells isolated from PBMCs by magnetic beads. Ctrl wells (triangles) represent cells without supernatants or pellets for each experiment. There was reduced IL-27 expression when the supernatants from mice that had been cultured with antibiotics were added to the CD11b+CD11c ${ }^{+}$cells. ${ }^{*} P=0.01$, ANOVA.

Serum cytokine measurements. Human cytokines were measured with an ultrasensitive Milliplex xMAP assay (Millipore). The lower limit of detection levels for these assays was $1 \mathrm{pg} / \mathrm{ml}$. The data were $\log$ transformed for statistical analysis.

Cultures with total fecal pellets, supernatants, or pellets of fecal samples from humanized mice. Freshly collected fecal pellets from humanized mice that had or had not been treated with antibiotics ( 5 mice each) were pooled, dissolved in sterile PBS, and heat inactivated for 30 minutes at $95^{\circ} \mathrm{C}$. The number of bacteria was determined by their optical density; $2.2 \times 10^{6}$ bacteria, their supernatants, or their pellets (following centrifugation at $6,797 \mathrm{~g}$ for 15 minutes) were used to stimulate $2 \times 10^{5}$ splenocytes or PBMCs from humans, cultured in $200 \mu 1$ RPMI 1640 media (Invitrogen) supplemented with 10\% FCS. After overnight culture or 5 days of culture, levels of cytokines in the culture supernatants were determined by a Milliplex xMAP assay. The samples were tested against a panel of 4 cell donors.

Cultures with human stool samples. Human stool samples were collected and frozen from hospitalized patients that had or had not been treated with antibiotics in the past 3 months. The samples were dissolved in PBS and heat inactivated as described above. Equal numbers of bacteria $\left(2.2 \times 10^{6}\right)$, determined by OD, were added to cultures.

Statistics. Unless indicated, all data are presented as the mean \pm SEM. In general, individual symbols represent individual mice from 3-4 independent experiments, each with 3-6 mice/group. Comparisons between groups were performed with the indicated statistical tests. All analyses were done with GraphPad Prism software (version 6.0). A $P$ value of less than 0.05 was considered statistically significant. Student's $t$ tests were 2 tailed.

Study approval. IACUC approval was obtained for all studies. Written informed consent was given for donation of stool samples. The studies with human samples were approved by the IRBs at Yale University, New Haven, CT and the Albert Einstein College of Medicine, Bronx, NY.

\section{Author contributions}

EG, NKV, MM, SM, and $\mathrm{KCH}$ performed experiments, analyzed data, and wrote the manuscript. SD, JCR, and RT performed experiments and collected data. PPH and BL performed experiments and analyzed data, analyzed data, and wrote the manuscript, RT collected and analyzed data. SMV, JDHM, MK, ALG, BCH, and CC analyzed data and wrote the manuscript.

\section{Acknowledgments}

This work was supported by grants U01AI102011, R01DK057846, and T35HL007649 from the NIH. 
Address correspondence to: Kevan C. Herold, Department of Immunobiology and Internal Medicine, Yale University, 300 George Street 353E, New Haven, Connecticut 06520, USA. Phone: 203.785.6507; Email: kevan.herold@yale.edu.

1. Maynard CL, Elson CO, Hatton RD, Weaver CT. Reciprocal interactions of the intestinal microbiota and immune system. Nature. 2012;489(7415):231-241.

2. Lee YK, Mazmanian SK. Has the microbiota played a critical role in the evolution of the adaptive immune system? Science. 2010;330(6012):1768-1773.

3. Coyte KZ, Schluter J, Foster KR. The ecology of the microbiome: Networks, competition, and stability. Science. 2015;350(6261):663-666.

4. Belkaid Y, Hand TW. Role of the microbiota in immunity and inflammation. Cell. 2014;157(1):121-141.

5. Hall JA, et al. Commensal DNA limits regulatory $\mathrm{T}$ cell conversion and is a natural adjuvant of intestinal immune responses. Immunity. 2008;29(4):637-649.

6. Ochoa-Repáraz J, et al. Role of gut commensal microflora in the development of experimental autoimmune encephalomyelitis. J Immunol. 2009;183(10):6041-6050.

7. Round JL, Mazmanian SK. Inducible Foxp3 $3^{+}$regulatory T-cell development by a commensal bacterium of the intestinal microbiota. Proc Natl Acad Sci USA. 2010;107(27):12204-12209.

8. Macho Fernandez E, et al. Anti-inflammatory capacity of selected lactobacilli in experimental colitis is driven by NOD2-mediated recognition of a specific peptidoglycan-derived muropeptide. Gut. 2011;60(8):1050-1059.

9. Kim KS, et al. Dietary antigens limit mucosal immunity by inducing regulatory $\mathrm{T}$ cells in the small intestine. Science. 2016;351(6275):858-863.

10. Hand TW, et al. Acute gastrointestinal infection induces long-lived microbiota-specific T cell responses. Science. 2012;337(6101):1553-1556.

11. Vaarala O. Human intestinal microbiota and type 1 diabetes. Curr Diab Rep. 2013;13(5):601-607.

12. Kostic $\mathrm{AD}$, et al. The dynamics of the human infant gut microbiome in development and in progression toward type 1 diabetes. Cell Host Microbe. 2015;17(2):260-273.

13. Vatanen T, et al. Variation in microbiome LPS immunogenicity contributes to autoimmunity in humans. Cell. 2016;165(4):842-853.

14. Vétizou M, et al. Anticancer immunotherapy by CTLA-4 blockade relies on the gut microbiota. Science. 2015;350(6264):1079-1084

15. Viaud S, et al. The intestinal microbiota modulates the anticancer immune effects of cyclophosphamide. Science. 2013;342(6161):971-976.

16. Sivan A, et al. Commensal Bifidobacterium promotes antitumor immunity and facilitates anti-PD-L1 efficacy. Science. 2015;350(6264):1084-1089.

17. Waldron-Lynch F, et al. Teplizumab induces human gut-tropic regulatory cells in humanized mice and patients. Sci Transl Med. 2012;4(118):118ra12.

18. Bisikirska B, Colgan J, Luban J, Bluestone JA, Herold KC. TCR stimulation with modified anti-CD3 mAb expands CD $8^{+} \mathrm{T}$ cell population and induces CD8 ${ }^{+}$CD25+ Tregs. J Clin Invest. 2005;115(10):2904-2913.

19. Herold KC, Burton JB, Francois F, Poumian-Ruiz E, Glandt M, Bluestone JA. Activation of human T cells by FcR nonbinding anti-CD3 mAb, hOKT3gamma1(Ala-Ala). J Clin Invest. 2003;111(3):409-418.

20. Tooley JE, et al. Changes in T-cell subsets identify responders to FcR-nonbinding anti-CD3 mAb (teplizumab) in patients with type 1 diabetes. Eur J Immunol. 2016;46(1):230-241.

21. Kaech SM, Tan JT, Wherry EJ, Konieczny BT, Surh CD, Ahmed R. Selective expression of the interleukin 7 receptor identifies effector CD8 T cells that give rise to long-lived memory cells. Nat Immunol. 2003;4(12):1191-1198.

22. Herold KC, et al. Teplizumab treatment may improve C-peptide responses in participants with type 1 diabetes after the newonset period: a randomised controlled trial. Diabetologia. 2013;56(2):391-400.

23. Esplugues E, et al. Control of TH17 cells occurs in the small intestine. Nature. 2011;475(7357):514-518.

24. Takiishi $\mathrm{T}$, et al. Reversal of autoimmune diabetes by restoration of antigen-specific tolerance using genetically modified Lactococcus lactis in mice. J Clin Invest. 2012;122(5):1717-1725.

25. Zhu C, et al. An IL-27/NFIL3 signalling axis drives Tim-3 and IL-10 expression and T-cell dysfunction. Nat Commun. 2015;6:6072.

26. Hall AO, et al. The cytokines interleukin 27 and interferon- $\gamma$ promote distinct Treg cell populations required to limit infectioninduced pathology. Immunity. 2012;37(3):511-523.

27. Dixon KO, van der Kooij SW, Vignali DA, van Kooten C. Human tolerogenic dendritic cells produce IL-35 in the absence of other IL-12 family members. Eur J Immunol. 2015;45(6):1736-1747.

28. Saito H, Tsurikisawa N, Oshikata C, Tsuburai T, Akiyama K. Increased interleukin-27 production by antigen-presenting cells promotes regulatory $\mathrm{T}$ cell differentiation and contributes to inducing a remission in patients with eosinophilic granulomatosis with polyangiitis. Int Arch Allergy Immunol. 2013;161 Suppl 2:66-74.

29. Wu HY, et al. In vivo induction of Tr1 cells via mucosal dendritic cells and AHR signaling. PLoS ONE. 2011;6(8):e23618.

30. Vudattu NK, et al. Humanized mice as a model for aberrant responses in human T cell immunotherapy. J Immunol. 2014;193(2):587-596.

31. Herold KC, Taylor L. Treatment of Type 1 diabetes with anti-CD3 monoclonal antibody: induction of immune regulation? Immunol Res. 2003;28(2):141-150.

32. Zhang N, et al. Regulatory $\mathrm{T}$ cells sequentially migrate from inflamed tissues to draining lymph nodes to suppress the alloimmune response. Immunity. 2009;30(3):458-469.

33. Baatar D, Olkhanud P, Sumitomo K, Taub D, Gress R, Biragyn A. Human peripheral blood T regulatory cells (Tregs), functionally primed CCR4 ${ }^{+}$Tregs and unprimed CCR4- Tregs, regulate effector T cells using FasL. J Immunol. 2007;178(8):4891-4900.

34. Lee I, Wang L, Wells AD, Dorf ME, Ozkaynak E, Hancock WW. Recruitment of Foxp $3^{+}$T regulatory cells mediating allograft 
tolerance depends on the CCR4 chemokine receptor. J Exp Med. 2005;201(7):1037-1044.

35. Chang PV, Hao L, Offermanns S, Medzhitov R. The microbial metabolite butyrate regulates intestinal macrophage function via histone deacetylase inhibition. Proc Natl Acad Sci USA. 2014;111(6):2247-2252.

36. Alenghat T, Artis D. Epigenomic regulation of host-microbiota interactions. Trends Immunol. 2014;35(11):518-525.

37. Jeon SG, et al. Probiotic Bifidobacterium breve induces IL-10-producing Tr1 cells in the colon. PLoS Pathog. 2012;8(5):e1002714.

38. Smith PM, et al. The microbial metabolites, short-chain fatty acids, regulate colonic Treg cell homeostasis. Science. 2013;341(6145):569-573.

39. Furusawa Y, et al. Commensal microbe-derived butyrate induces the differentiation of colonic regulatory T cells. Nature. 2013;504(7480):446-450.

40. Singh N, et al. Activation of Gpr109a, receptor for niacin and the commensal metabolite butyrate, suppresses colonic inflammation and carcinogenesis. Immunity. 2014;40(1):128-139.

41. Watson DW, Kim YB. Modification of host responses to bacterial endotoxins. I. Specificity of pyrogenic tolerance and the role of hypersensitivity in pyrogenicity, lethality, and skin reactivity. J Exp Med. 1963;118:425-446.

42. Muzio M, et al. Differential expression and regulation of toll-like receptors (TLR) in human leukocytes: selective expression of TLR3 in dendritic cells. J Immunol. 2000;164(11):5998-6004.

43. Bach JF. The effect of infections on susceptibility to autoimmune and allergic diseases. N Engl J Med. 2002;347(12):911-920.

44. Cullen TW, et al. Gut microbiota. Antimicrobial peptide resistance mediates resilience of prominent gut commensals during inflammation. Science. 2015;347(6218):170-175.

45. Kozich JJ, Westcott SL, Baxter NT, Highlander SK, Schloss PD. Development of a dual-index sequencing strategy and curation pipeline for analyzing amplicon sequence data on the MiSeq Illumina sequencing platform. Appl Environ Microbiol. 2013;79(17):5112-5120.

46. McDonald D, et al. An improved Greengenes taxonomy with explicit ranks for ecological and evolutionary analyses of bacteria and archaea. ISME J. 2012;6(3):610-618. 